\title{
Application of chiral 2-isoxazoline for the synthesis of syn-1,3-diol analogs
}

\author{
Juanjuan Feng, Tianyu Li, Jiaxin Zhang ${ }^{*}$ and Peng Jiao*
}

\author{
Letter \\ Address: \\ Key Laboratory of Radiopharmaceuticals, College of Chemistry, \\ Beijing Normal University, Beijing 100875, P.R. China \\ Email: \\ Jiaxin Zhang* - zhangjiaxin@bnu.edu.cn; Peng Jiao* - \\ pjiao@bnu.edu.cn \\ * Corresponding author \\ Keywords: \\ $\beta$-hydroxy ketone; cycloaddition; 1,3-diol; isoxazoline; silyl nitronate
}

\author{
Beilstein J. Org. Chem. 2019, 15, 1840-1847. \\ doi:10.3762/bjoc. 15.179 \\ Received: 17 April 2019 \\ Accepted: 12 July 2019 \\ Published: 01 August 2019 \\ Associate Editor: T. P. Yoon \\ (C) 2019 Feng et al.; licensee Beilstein-Institut. \\ License and terms: see end of document.
}

\section{Abstract}

The asymmetric cycloaddition of TIPS nitronate catalyzed by " $\mathrm{Cu}$ (II)-bisoxazoline" gave the 2-isoxazoline product in $95 \%$ yield, which was converted into tert-butyl $(3 S, 5 R)$-6-hydroxy-3,5-O-isopropylidene-3,5-dihydroxyhexanoate in 14 steps through a $\beta$-hydroxy ketone.

\section{Introduction}

The chiral 1,3-diol structure is widespread in a broad spectrum of natural products $[1,2]$. $(3 R)-\beta$-Hydroxy- $\delta$-lactone or its openring equivalent (3R)-syn-3,5-dihydroxypentanoic acid, is a common structure in naturally occurring mevastatin (or compactin), lovastatin or closely related statins, and synthetic statins. Either the syn or anti-1,3-diol could be prepared from enantiomerically pure $\beta$-hydroxy ketones through $\beta$-hydroxydirected carbonyl reduction following Evans' [3] or Prasad's [4-11] method. The Narasaka-Prasad reduction of a $\delta$-hydroxy$\beta$-keto esters derived from $\beta$-hydroxy esters [12-23] is widely used to prepare tert-butyl (3R)-3,5-O-isopropylidene-3,5-dihydroxyhexanoate (Scheme 1a) [24-37], which is a building block for synthetic statins [38-41], though enzymatic syntheses [42$48]$ of the chiral $\beta$-hydroxy- $\delta$-lactone moiety or its equivalents, pioneered by Wong [42], is equally competitive. Here, we report the preparations of tert-butyl $(3 S, 5 R)$-6-hydroxy-3,5-Oisopropylidene-3,5-dihydroxyhexanoate and related syn-1,3-diol analogs from a chiral 2-isoxazoline (Scheme 1b). This work is part of our continuous efforts in asymmetric syntheses and applications of chiral 2-isoxazolines [49-51].

\section{Results and Discussion}

Our synthesis commenced with a chiral 3,5-disubstituted-2isoxazoline $\mathbf{3}$ or $\mathbf{4}$, which were prepared from silyl nitronate through an asymmetric 1,3-dipolar cycloaddition developed in our lab (Table 1) [49]. The synthesis of the triisopropylsilyl nitronate was initially attempted starting with 3-nitropropionic acid methyl ester but no desired product was observed. How- 
(a)<smiles>[R]CC1C[C@@H](CC(=O)OC(C)(C)C)OC(C)(C)O1</smiles><smiles>CC(=O)OC(C)(C)C</smiles><smiles>CCOC(=O)CC(=O)CC(O)C(=O)OCC</smiles>

(b)

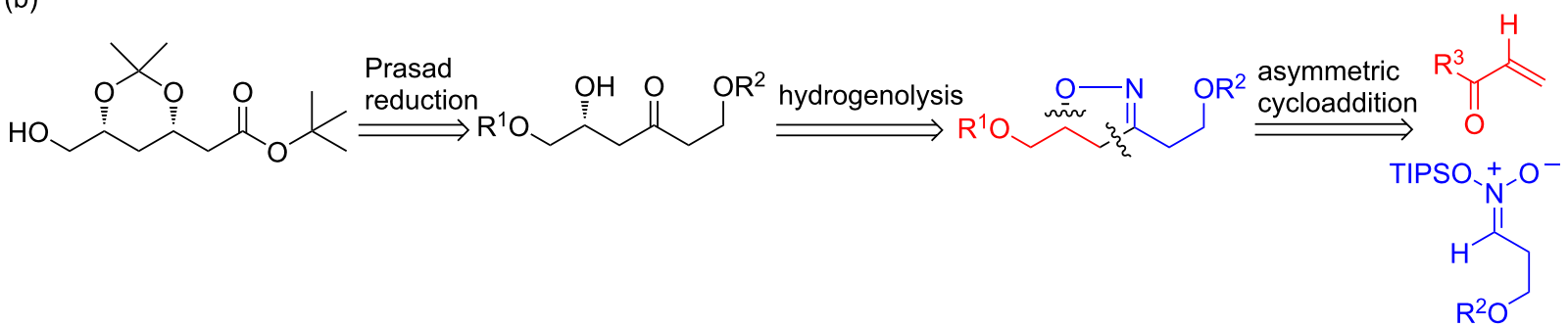

Scheme 1: Accesses to tert-butyl 3,5-O-isopropylidene-3,5-dihydroxyhexanoates. (a) Previous methods using Claisen condensation. (b) Our new method using cycloaddition.

Table 1: Optimization of the conditions for the asymmetric cycloaddition.

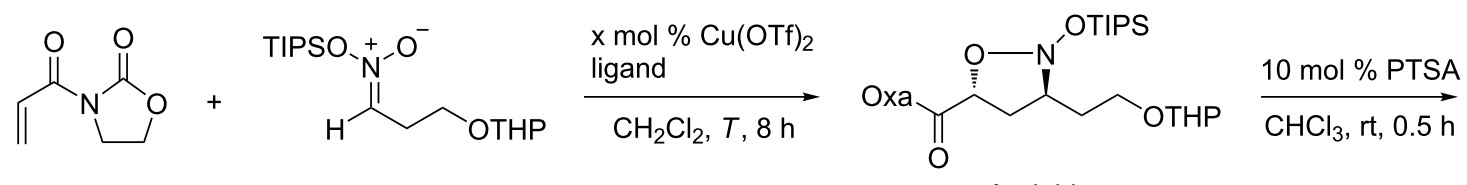

1 , yield

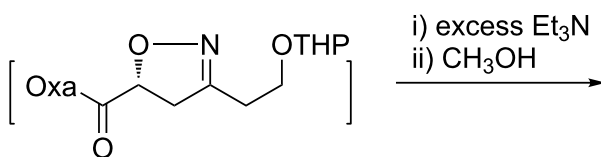<smiles>CC(=O)C1CC(CC[OH+])=NO1</smiles>

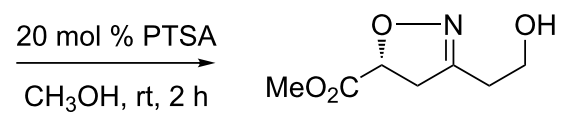

4, ee ee $(\%)(4)$

\begin{tabular}{cccccc}
\hline Entry & Ligand & $\mathrm{x} \mathrm{mol} \% \mathrm{Cu}$ & $T\left({ }^{\circ} \mathrm{C}\right)$ & Yield (\%) (1) & ee (\%) (4) \\
\hline 1 & A & 10 & -50 & 25 & 18 \\
2 & $\mathrm{~A}$ & 20 & -50 & 75 & 72 \\
3 & $\mathrm{~B}$ & 10 & -50 & 70 & 86 \\
4 & $\mathrm{~B}$ & 20 & -50 & 95 & 78 \\
5 & $\mathrm{~B}$ & 20 & -40 & 84 & 80 \\
6 & $\mathrm{~B}$ & 20 & -60 & & \\
\hline
\end{tabular}<smiles>CC(C)(C1=N[C@@H](C(C)(C)C)CO1)C1=N[C@H](C(C)(C)C)CO1</smiles>

ligand $\mathrm{A}$<smiles>CC(C)[C@H]1COC(C(C)(C)C2=N[C@H](C(C)C)CO2)=N1</smiles>

ligand $B$ ever, switching to 3-nitropropanol, protected as the THP ether, succeeded to prepare the required triisopropylsilyl nitronate. Then, the catalytic asymmetric cycloaddition gave the 2 -isoxazolidine cycloadduct $\mathbf{1}$ in a high yield. In the light of our previous ligand screening results [49], two bisoxazolines with an isopropyl (ligand B) or tert-butyl group (ligand A) were tested. Optimization of the conditions established that $26 \mathrm{~mol} \%$ of ligand $\mathrm{B}$ together with $20 \mathrm{~mol} \% \mathrm{Cu}(\mathrm{OTf})_{2}$ in anhydrous 
$\mathrm{CH}_{2} \mathrm{Cl}_{2}$ catalyzed the cycloaddition between $\mathrm{N}$-acryloyl-1,3oxazolidin-2-one and the silyl nitronate at $-50{ }^{\circ} \mathrm{C}$ to give $\mathbf{1}$ in $95 \%$ isolated yield, which subsequently generated 3,5-disubstituted isoxazoline 4 in $80 \%$ ee. Decreasing the amount of the chiral Lewis acid catalyst led to a decrease of both the ee and the yield. Desilylation of the 2-isoxazolidine 1 was effected in $\mathrm{CHCl}_{3}$ using catalytic amounts of $p$-toluenesulfonic acid (PTSA). Though the yield of the in situ-generated 2-isoxazoline 2 bearing the 1,3-oxazolidin-2-one auxiliary was perfect, purification of $\mathbf{2}$ by silica gel chromatography was problematic due to decomposition. No pure product was isolated from crude 2 by chromatography on silica gel. Decomposition occurred to a compound similar to 2 , in which the 3 -substituent was $\mathrm{CH}_{2} \mathrm{OH}$ [49]. To overcome this problem, the crude reaction mixture containing 2 and PTSA was concentrated before excess $\mathrm{Et}_{3} \mathrm{~N}$ was added followed by $\mathrm{CH}_{3} \mathrm{OH}$ as the solvent. These operations removed the 1,3-oxazolidin-2-one auxiliary while preserving the THP group, and afforded the corresponding methyl ester $\mathbf{3}$ (Table 1), which was stable and could be subjected to silica gel chromatography. Compound $\mathbf{4}$ was used to determine the stereoselectivity of the cycloaddition step as well as for oxidation.

Oxidation of the 2-isoxazoline 4 with Jones' reagent gave a complicated mixture, in which the desired carboxylic acid was not observed (Scheme 2). The stepwise oxidation of the free hydroxy to the carboxy group via intermediary aldehyde was then examined. Swern or pyridinium chlorochromate (PCC) oxidation of $\mathbf{4}$ also gave a complicated mixture without the desired aldehyde detected. These failed reactions indicated that the 2-isoxazoline moiety could not survive oxidation conditions. Based on this assumption, the corresponding silyl nitronate from 3-nitropropanal or its acetal were not tried for cycloaddition.

We then set to liberate the $\beta$-hydroxy ketone synthon by ring opening of the isoxazoline 3 (Scheme 3). Raney-Ni-catalyzed hydrogenolysis in the presence of boronic acid had been widely utilized to disconnect the $\mathrm{N}-\mathrm{O}$ bond as well as to hydrolyze the resulting imine into a ketone [52]. We applied this method to deprotect the isoxazoline 3. However, the desired $\beta$-hydroxy ketone was never obtained. In one instance, the methyl ketone from a retro-aldol reaction of the desired $\beta$-hydroxy ketone was observed. In our experience, the hydrogenolysis of a 2-isoxazoline having a 5-ester group was troublesome. Thus, the 5-ester group was reduced with $\mathrm{NaBH}_{4}$ to give 5. The hydroxy group was subsequently protected with benzoyl (Scheme 3), which also worked as a chromophore facilitating HPLC analysis. Afterwards, we tried oxidations once again. After removal of THP from 6 , the resulting compound 6 ' was subjected to oxidation with various reagents (Scheme 4) [5355]. The expected carboxylic acid or aldehyde was not observed, which further verified the intolerance exemplified in Scheme 2 . These results prompted us to try the oxidation in a later stage.

When 6 was subjected to Raney-Ni-catalyzed hydrogenolysis, the desired $\beta$-hydroxy ketone 7 was obtained in $85 \%$ yield (Scheme 3). Under the weakly acidic conditions, the THP group survived. Next, a Narasaka-Prasad reduction [4-11] of 7 using $\mathrm{Et}_{2} \mathrm{BOMe}$ and $\mathrm{NaBH}_{4}$ at $-78{ }^{\circ} \mathrm{C}$ gave stable ethylboronate 8 in $96 \%$ yield. Several ethylboronate compounds have been reported [9-11,56-62]. From 8 to 9 , no $\mathrm{H}_{2} \mathrm{O}_{2}$ treatment was necessary. Rotary evaporation of 8 with $\mathrm{CH}_{3} \mathrm{OH}$ at ca. $40{ }^{\circ} \mathrm{C}$ easily removed the ethylborane group. Removal of THP in 9 delivered a 1,3,5-trihydroxy compound 10. In another way, $\mathbf{1 0}$ could be prepared by treating 8 with PTSA in $\mathrm{CH}_{3} \mathrm{OH}$ at rt. NMR spectra of 8-10 exhibited only one set of signals corresponding to the syn-dihydroxy products, indicating an extra high diastereoselectivity (syn:anti $>99: 1$ ) during the reduction. To unambiguously determine the diastereomeric ratio, the anti-1,3diol corresponding to $\mathbf{1 0}$ was prepared from 7 by $\mathrm{RuCl}_{3}-\mathrm{PPh}_{3}-$ catalyzed hydrogenation $[63,64]$. However, the two diastereomers had identical proton NMR spectra.

The terminal hydroxy group of $\mathbf{1 0}$ was protected with TBS [6569] and the syn-hydroxy groups subjected to acetonization using PTSA and dimethoxypropane (DMP) to give $\mathbf{1 2}$ in $86 \%$ total yield [70]. Treatment of $\mathbf{1 2}$ with TBAF again liberated the terminal hydroxy group for further oxidation. $\mathrm{RuCl}_{3}$-catalyzed

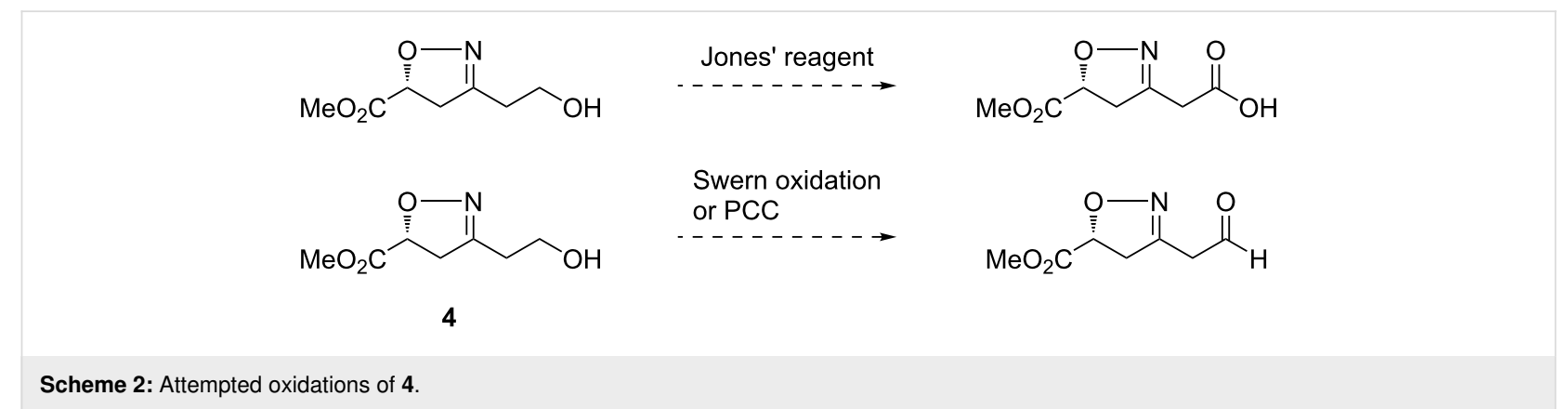




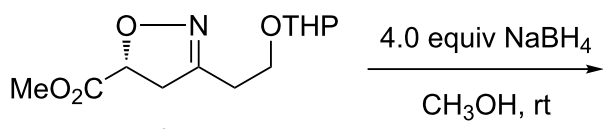

3

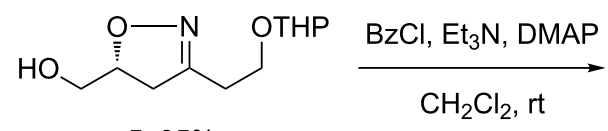

5, $95 \%$<smiles>O=C(OCc1ccccc1)OCC1CC(CC[OH+])ON1</smiles>

6, $97 \%$

$$
\begin{gathered}
10 \text { equiv } \mathrm{B}(\mathrm{OH})_{3} \\
\text { Raney Ni} \\
1 \text { atm } \mathrm{H}_{2} \\
\underset{\substack{\mathrm{H}_{2} \mathrm{O} / \mathrm{CH}_{3} \mathrm{OH} \\
\mathrm{rt}, 2 \mathrm{~h}}}{\longrightarrow}
\end{gathered}
$$<smiles>O=C(CC[OH+])C[C@@H](O)COC(=O)O</smiles>

$7,85 \%$

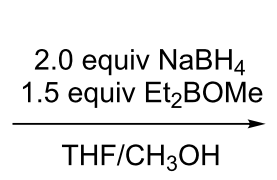

$-78^{\circ} \mathrm{C}, 1 \mathrm{~h}$

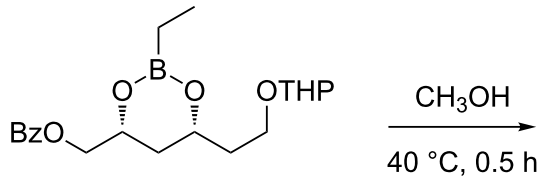

8, $96 \%$

cis: trans $>99: 1$ $40{ }^{\circ} \mathrm{C}, 0.5 \mathrm{~h}$

\section{2 equiv TBSCl}

2.0 equiv $\mathrm{Et}_{3} \mathrm{~N}$

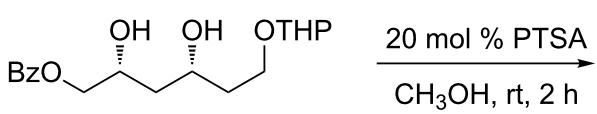

9, $99 \%$

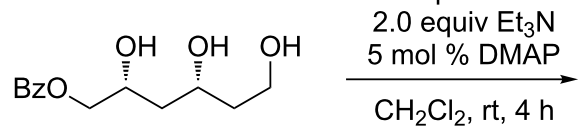<smiles>OC(CO[SbH3])C[C@@H](O)CC[OH+]</smiles>

$10,89 \%$

$11,87 \%$

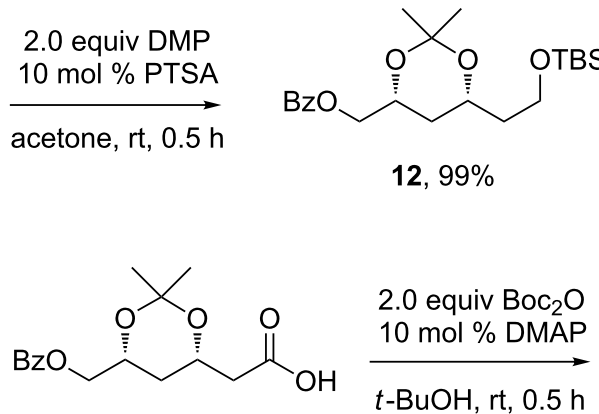

$14,86 \%$

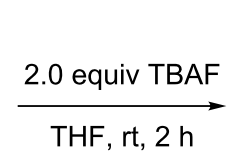<smiles>CC(C)(C)OC(=O)C[C@@H]1C[C@H](COC(=O)c2ccccc2)OC(C)(C)O1</smiles>

15<smiles>CC1(C)O[C@@H](CCO)C[C@H](COC(=O)c2ccccc2)O1</smiles>

$13,99 \%$
3.0 equiv $\mathrm{NaIO}_{4}$ $\underset{\mathrm{CCl}_{4} / \mathrm{CH}_{3} \mathrm{CN} / \mathrm{H}_{2} \mathrm{O}}{\stackrel{10 \mathrm{~mol} \% \mathrm{RuCl}_{3}}{\longrightarrow}}$ $\mathrm{rt}, 0.5 \mathrm{~h}$ $86 \%$ yield, $74 \%$ ee

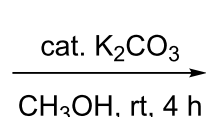<smiles>CC(C)(C)OC(=O)C[C@H]1CC(CO)OC(C)(C)O1</smiles>

$16,87 \%$

$\underset{\mathrm{CH}_{2} \mathrm{Cl}_{2}, \mathrm{rt}, 0.5 \mathrm{~h}}{\stackrel{1.2 \text { equiv } \mathrm{Ac}_{2} \mathrm{O}}{2.5 \text { equiv } \mathrm{Et}_{3} \mathrm{~N}}}$<smiles>CC(=O)OC[C@H]1C[C@@H](CC(=O)OC(C)(C)C)OC(C)(C)O1</smiles>

$17,99 \%$<smiles>O=C(O)CC1CC(CCO)=NO1</smiles><smiles>O=C(OCC1CC(CCO)=NO1)c1ccccc1</smiles>

6'
Jones' reagent

$\mathrm{NaClO}_{2} /$ TEMPO or

the method of $\mathrm{Ma}$

Dess-Martin<smiles>O=C(O)CC1=NOC(COC(=O)c2ccccc2)C1</smiles><smiles>O=CCC1=NO[C@@H](COC(=O)c2ccccc2)C1</smiles> 
oxidation of 13 with $\mathrm{NaIO}_{4}$ yielded the carboxylic acid 14 in $86 \%$ yield [70], which was reacted with $\mathrm{Boc}_{2} \mathrm{O}$ to get the tertbutyl ester $\mathbf{1 5}$ [26,43,71]. The ee of $\mathbf{1 5}$ was determined as $74 \%$. The racemic sample of $\mathbf{1 5}$ was prepared from racemic diethyl malate following known methods [26,27]. Finally, $\mathrm{K}_{2} \mathrm{CO}_{3}$-catalyzed methanolysis gave $\mathbf{1 6}$ in $87 \%$ yield [26,27]. The absolute stereochemistry of $\mathbf{1 6}$ was confirmed by crystal structure analysis [72] and the specific rotation [28] of 17. Centimeter-long prismatic single crystals of $\mathbf{1 7}$ were obtained by slow evaporation of a petroleum solution.

Starting from 9, we tested several reactions in order to selectively protect the internal hydroxy groups (Scheme 5). Though not fruitful, these results deserve some comments. The PTSAcatalyzed acetonization of 9 using 2.0 equiv DMP gave the acetonide 18 in a quantitative yield. Treating 18 with a catalytic amount of PTSA in methanol gave 10, with the protecting groups removed except benzoyl. PTSA-catalyzed acetonization of $\mathbf{1 0}$ using 2.0 equiv DMP gave a mixture of two acetonides $\mathbf{1 9}$ and $\mathbf{1 3}$, which are separable by silica gel chromatography (Scheme 5a). In another trial (Scheme 5b), acylation of the two hydroxy groups in $\mathbf{9}$ yielded $\mathbf{2 0}$ in a quantitative yield. PTSAcatalyzed removal of THP in $\mathbf{2 0}$ in methanol did occur. However, concomitant monodeacylation as well as further an acyltransfer reaction also took place, resulting in a mixture. These results indicated THP, isopropylidene or Ac protection to primary or secondary hydroxy groups did not well tolerate PTSAcatalyzed methanolysis.

\section{Conclusion}

In conclusion, we synthesized tert-butyl (3S,5R)-6-hydroxy-3,5$O$-isopropylidene-3,5-dihydroxyhexanoate (16), which is enantiomeric to a key intermediate for atorvastatin, from a chiral 2 -isoxazoline (3). The $\beta$-hydroxy ketone $\mathbf{7}$ obtained from $\mathbf{3}$ could be easily converted into several syn-1,3-diol analogs, demonstrating the usefulness of chiral 2-isoxazolines.

\section{Experimental}

1: To a dry Schlenk tube were added $\mathrm{Cu}(\mathrm{OTf})_{2}(144 \mathrm{mg}$, $0.4 \mathrm{mmol})$, chiral bisoxazoline $\mathrm{B}(139 \mathrm{mg}, 0.52 \mathrm{mmol})$ and anhydrous $\mathrm{CH}_{2} \mathrm{Cl}_{2}(4 \mathrm{~mL})$ under $\mathrm{N}_{2}$. After stirring at room temperature for $2 \mathrm{~h}$, a clear solution had formed, which was cooled to $-50{ }^{\circ} \mathrm{C}$ and $\mathrm{N}$-acryloyl-1,3-oxazolidin-2-one (282 $\mathrm{mg}$, $2 \mathrm{mmol}$ ) was added. After stirring for $30 \mathrm{~min}$, a solution of the silyl nitronate $(3.0 \mathrm{mmol})$ in anhydrous $\mathrm{CH}_{2} \mathrm{Cl}_{2}(6 \mathrm{~mL})$ was added. The mixture was stirred for $8 \mathrm{~h}$ at $-50{ }^{\circ} \mathrm{C}$ and monitored by TLC. After the reaction was completed, the product was purified by silica gel chromatography. Yellow oil (923 mg, 95\% yield); $R_{\mathrm{f}} 0.40$ (1:1 hexanes/AcOEt); ${ }^{1} \mathrm{H}$ NMR (400 MHz, $\left.\mathrm{CDCl}_{3}\right) \delta 5.77-5.74\left(\mathrm{~m}, 1 \mathrm{H}, \mathrm{CH}_{2} \mathrm{CHO}\right), 4.53(\mathrm{~s}, 1 \mathrm{H}, \mathrm{OCHO})$,

(a)<smiles>[R6]OC[C@H](O)C[C@H](O)CC[OH+]</smiles>

9

\section{$10 \mathrm{~mol} \%$ PTSA}

2.0 equiv DMP

acetone, rt, $0.5 \mathrm{~h}$

$\mathrm{BzO}$<smiles>CC(=O)C[C@H]1C[C@@H](CC[O-])OC(C)(C)O1</smiles>

18, $99 \%$<smiles>O=C(OC[C@@H](O)C[C@@H](O)CCO)c1ccccc1</smiles>

10, $89 \%$

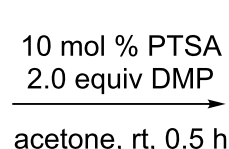

acetone, $\mathrm{rt}, 0.5 \mathrm{~h}$

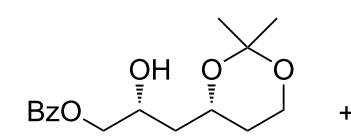

$19,54 \%$<smiles>CC1(C)O[C@@H](CCO)C[C@H](COC(=O)OCc2ccccc2)O1</smiles>

$13,30 \%$

(b)

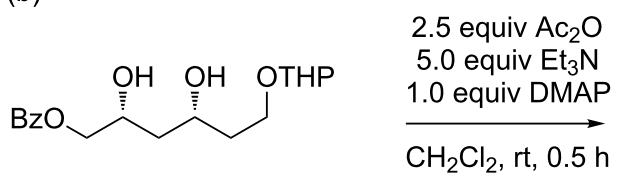

9

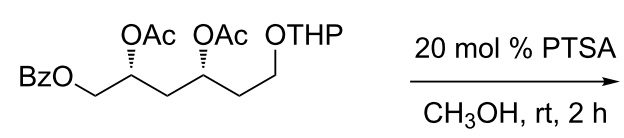

$20,99 \%$

CH, 2 h

\section{$\underset{\mathrm{CH}_{3} \mathrm{OH}, \mathrm{rt}, 2 \mathrm{~h}}{\stackrel{20 \mathrm{~mol} \% \mathrm{PTSA}}{\longrightarrow}}$}

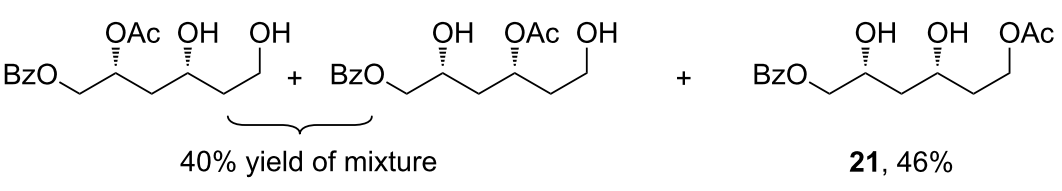

Scheme 5: Attempted selective protections of internal 1,3-hydroxy groups: (a) acetonizations of 1,3-diols; (b) removal of co-existing Ac and THP on hydroxy groups. 
$4.44\left(\mathrm{t}, J=8.0 \mathrm{~Hz}, 2 \mathrm{H}, \mathrm{CH}_{2} \mathrm{O}\right), 4.03-3.99\left(\mathrm{~m}, 2 \mathrm{H}, \mathrm{CH}_{2} \mathrm{O}\right)$, 3.79-3.74 (m, 2H, $\left.\mathrm{OCH}_{2} \mathrm{CH}_{2}\right), 3.47-3.37(\mathrm{~m}, 3 \mathrm{H}, \mathrm{NCH}$ and $\left.\mathrm{NCH}_{2}\right), 2.75-2.66\left(\mathrm{~m}, 1 \mathrm{H}, \mathrm{CHCH}_{2} \mathrm{CH}\right), 2.31-2.27(\mathrm{~m}, 1 \mathrm{H}$, $\left.\mathrm{CHCH}_{2} \mathrm{CH}\right), 2.17-2.12\left(\mathrm{~m}, 1 \mathrm{H}, \mathrm{CH}_{2} \mathrm{CH}_{2}\right), 1.84-1.79(\mathrm{~m}, 2 \mathrm{H}$, $\mathrm{CH}_{2} \mathrm{CH}_{2}$ and $\left.\mathrm{CH}_{2} \mathrm{CH}_{2} \mathrm{CH}_{2}\right), 1.68-1.49\left(\mathrm{~m}, 6 \mathrm{H}, \mathrm{CH}_{2} \mathrm{CH}_{2} \mathrm{CH}_{2}\right)$, 1.24-1.15 (m, 3H, $\mathrm{SiCH}), 1.07-1.01\left(\mathrm{~m}, 18 \mathrm{H}, \mathrm{SiCH}\left(\mathrm{CH}_{3}\right)_{2}\right)$; ${ }^{13} \mathrm{C}$ NMR (100 MHz, $\left.\mathrm{CDCl}_{3}\right) \delta 170.8,153.1,98.9,98.9,77.4$, $77.2,69.9,69.8,65.2,62.8,62.5,62.3,42.6,35.6,35.5,30.7$, $30.6,29.9,29.8,25.5,19.6,19.5,18.1,18.0,12.2 ; \mathrm{IR}\left(\mathrm{cm}^{-1}\right)$ : 3544, 2942, 2867, 2725, 2249, 1780, 1704, 1464, 1386, 1275, 1133, 1035, 883, 806, 677; ESIMS $(\mathrm{m} / \mathrm{z}):[\mathrm{M}+\mathrm{Na}]^{+}$calcd for $\mathrm{C}_{23} \mathrm{H}_{42} \mathrm{~N}_{2} \mathrm{O}_{7} \mathrm{Si}$, 509.2659; found, 509.2659.

3: To a solution of $\mathbf{1}(0.86 \mathrm{~g}, 1.78 \mathrm{mmol})$ in $\mathrm{CHCl}_{3}(15 \mathrm{~mL})$ was added PTSA $(31 \mathrm{mg}, 0.178 \mathrm{mmol})$ at $0{ }^{\circ} \mathrm{C}$. The mixture was allowed to warm to room temperature and stirred until complete consumption of the starting material $(0.5 \mathrm{~h})$. Vacuum was applied to remove the solvent before $\mathrm{Et}_{3} \mathrm{~N}(5 \mathrm{~mL})$ was added. After stirring for $5 \mathrm{~min}$, methanol $(30 \mathrm{~mL})$ was added and the mixture was stirred overnight at room temperature. The crude product was purified by column chromatography. Yellow oil $(0.41 \mathrm{~g}, 89 \%$ yield $) ; R_{\mathrm{f}} 0.42$ (1:1 hexanes/AcOEt); ${ }^{1} \mathrm{H}$ NMR $\left(400 \mathrm{MHz}, \mathrm{CDCl}_{3}\right) \delta 4.92-4.87(\mathrm{~m}, 1 \mathrm{H}, \mathrm{OCHCO}), 4.51-4.50$ (m, $1 \mathrm{H}, \mathrm{OCHO}), 3.88-3.82\left(\mathrm{~m}, 1 \mathrm{H}, \mathrm{CH}_{2} \mathrm{O}\right), 3.75-3.71(\mathrm{~m}, 1 \mathrm{H}$, $\left.\mathrm{CH}_{2} \mathrm{O}\right), 3.68\left(\mathrm{~s}, 3 \mathrm{H}, \mathrm{CH}_{3}\right), 3.56-3.50\left(\mathrm{~m}, 1 \mathrm{H}, \mathrm{CH}_{2} \mathrm{O}\right)$, $3.42-3.39\left(\mathrm{~m}, 1 \mathrm{H}, \mathrm{CH}_{2} \mathrm{O}\right), 3.24-3.31\left(\mathrm{~m}, 1 \mathrm{H}, \mathrm{CHCH}_{2} \mathrm{CH}\right)$, 2.62-2.54 (m, $\left.1 \mathrm{H}, \mathrm{CH}_{2} \mathrm{CH}_{2} \mathrm{CH}\right), 1.74-1.44(\mathrm{~m}, 6 \mathrm{H}$, $\left.\mathrm{CH}_{2} \mathrm{CH}_{2} \mathrm{CH}_{2}\right) ;{ }^{13} \mathrm{C}$ NMR (100 MHz, $\left.\mathrm{CDCl}_{3}\right) \delta 171.0,156.9$, 99.0, 98.9, 64.4, 64.3, 62.5, 62.4, 52.6, 41.6, 30.6, 27.9, 25.4, 19.6, 19.5; IR $\left(\mathrm{cm}^{-1}\right): 3481,2950,2873,2852,2657,1756$, 1738, 1734, 1628, 1456, 1436, 1367, 1354, 1201, 1134, 1034, 869, 814, 752, 740; $\operatorname{ESIMS~}(\mathrm{m} / \mathrm{z}):[\mathrm{M}+\mathrm{H}]^{+}$calcd for $\mathrm{C}_{12} \mathrm{H}_{19} \mathrm{NO}_{5}, 258.1341$; found, 258.1340.

\section{Supporting Information}

\section{Supporting Information File 1}

Experimental procedures and characterization data. [https://www.beilstein-journals.org/bjoc/content/ supplementary/1860-5397-15-179-S1.pdf]

\section{Supporting Information File 2}

Crystallographic data for $\mathbf{1 7 .}$

[https://www.beilstein-journals.org/bjoc/content/ supplementary/1860-5397-15-179-S2.cif]

\section{Acknowledgements}

Financial support from Beijing Normal University and the National Natural Science Foundation of China are acknowledged.

\section{ORCID ${ }^{\circledR}$ iDs}

Peng Jiao - https://orcid.org/0000-0003-4039-8300

\section{Preprint}

A non-peer-reviewed version of this article has been previously published as a preprint doi:10.3762/bxiv.2019.10.v1

\section{References}

1. Nicolaou, K. C.; Daines, R. A.; Ogawa, Y.; Chakraborty, T. K. J. Am. Chem. Soc. 1988, 110, 4696-4705. doi:10.1021/ja00222a030

2. Rychnovsky, S. D.; Griesegraber, G.; Kim, J. J. Am. Chem. Soc. 1994, 116, 2621-2622. doi:10.1021/ja00085a053 and references therein.

3. Evans, D. A.; Chapman, K. T.; Carreira, E. M. J. Am. Chem. Soc. 1988, 110, 3560-3578. doi:10.1021/ja00219a035

4. Narasaka, K.; Pai, H. C. Chem. Lett. 1980, 9, 1415-1418. doi:10.1246/cl.1980.1415

5. Narasaka, K.; Pai, F.-C. Tetrahedron 1984, 40, 2233-2238. doi:10.1016/0040-4020(84)80006-x

6. Kathawala, F. G.; Prager, B.; Prasad, K.; Repič, O.; Shapiro, M. J.; Stabler, R. S.; Widler, L. Helv. Chim. Acta 1986, 69, 803-805. doi:10.1002/hlca.19860690407

7. Chen, K.-M.; Hardtmann, G. E.; Prasad, K.; Repič, O.; Shapiro, M. J. Tetrahedron Lett. 1987, 28, 155-158. doi:10.1016/s0040-4039(00)95673-9

8. Chen, K.-M.; Gunderson, K. G.; Hardtmann, G. E.; Prasad, K.; Repic, O.; Shapiro, M. J. Chem. Lett. 1987, 16, 1923-1926. doi:10.1246/cl.1987.1923

9. Burova, S. A.; McDonald, F. E. J. Am. Chem. Soc. 2002, 124, 8188-8189. doi:10.1021/ja026255p

10. Walleser, P.; Brückner, R. Eur. J. Org. Chem. 2010, 4802-4822. doi:10.1002/ejoc.201000280

11. Becerril-Jiménez, F.; Ward, D. E. Org. Lett. 2012, 14, 1648-1651. doi:10.1021/ol300432y

12. Saito, S.; Hasegawa, T.; Inaba, M.; Nishida, R.; Fujii, T.; Nomizu, S.; Moriwake, T. Chem. Lett. 1984, 13, 1389-1392. doi:10.1246/cl.1984.1389

13. Saito, S.; Nagao, Y.; Miyazaki, M.; Inaba, M.; Moriwake, T. Tetrahedron Lett. 1986, 27, 5249-5252. doi:10.1016/s0040-4039(00)85181-3

14. Saito, S.; Ishikawa, T.; Kuroda, A.; Koga, K.; Moriwake, T. Tetrahedron 1992, 48, 4067-4086. doi:10.1016/s0040-4020(01)92187-8

15. Hamada, Y.; Yokokawa, F.; Kabeya, M.; Hatano, K.; Kurono, Y.; Shioiri, T. Tetrahedron 1996, 52, 8297-8306. doi:10.1016/0040-4020(96)00383-3

16. Sauret-Cladière, S.; Jeminet, G. Tetrahedron: Asymmetry 1997, 8 , 417-423. doi:10.1016/s0957-4166(96)00517-4

17. Nakatani, S.; Ikura, M.; Yamamoto, S.; Nishita, Y.; Itadani, S.; Habashita, H.; Sugiura, T.; Ogawa, K.; Ohno, H.; Takahashi, K.; Nakai, H.; Toda, M. Bioorg. Med. Chem. 2006, 14, 5402-5422. doi:10.1016/j.bmc.2006.03.032

18. Bode, M. L.; Gates, P. J.; Gebretnsae, S. Y.; Vleggaar, R. Tetrahedron 2010, 66, 2026-2036. doi:10.1016/j.tet.2010.01.043

19. Li, D.; Zhao, Y.; Ye, L.; Chen, C.; Zhang, J. Synthesis 2010, 3325-3331. doi:10.1055/s-0030-1258187

20. Kitamura, M.; Ohkuma, T.; Takaya, H.; Noyori, R. Tetrahedron Lett 1988, 29, 1555-1556. doi:10.1016/s0040-4039(00)80350-0 
21. Shao, L.; Kawano, H.; Saburi, M.; Uchida, Y. Tetrahedron 1993, 49, 1997-2010. doi:10.1016/s0040-4020(01)86300-6

22. Jeulin, S.; Duprat de Paule, S.; Ratovelomanana-Vidal, V.; Genêt, J.-P.; Champion, N.; Dellis, P. Angew. Chem., Int. Ed. 2004, 43, 320-325. doi:10.1002/anie.200352453

23. Qiu, L.; Wu, J.; Chan, S.; Au-Yeung, T. T.-L.; Ji, J.-X.; Guo, R.; Pai, C.-C.; Zhou, Z.; Li, X.; Fan, Q.-H.; Chan, A. S. C. Proc. Natl. Acad. Sci. U. S. A. 2004, 101, 5815-5820. doi:10.1073/pnas.0307774101

24. Beck, G.; Jendralla, H.; Kesseler, K. Synthesis 1995, 1014-1018. doi:10.1055/s-1995-4028

25. Nishiyama, A.; Horikawa, M.; Yasohara, Y.; Ueyama, N.; Inoue, K. Process for preparing optically active 2-[6-(hydroxymethyl)-1,3-dioxan-4-yl]acetic acid derivatives as pharmaceutical intermediates. WO Patent Appl. WO 2001094337 A1, Dec 13, 2001.

26. Szpilman, A. M.; Cereghetti, D. M.; Wurtz, N. R.; Manthorpe, J. M.; Carreira, E. M. Angew. Chem., Int. Ed. 2008, 47, 4335-4338. doi:10.1002/anie.200800589

27. Szpilman, A. M.; Cereghetti, D. M.; Manthorpe, J. M.; Wurtz, N. R.; Carreira, E. M. Chem. - Eur. J. 2009, 15, 7117-7128. doi:10.1002/chem.200900231

28. Fan, W.; Li, W.; Ma, X.; Tao, X.; Li, X.; Yao, Y.; Xie, X.; Zhang, Z. J. Org. Chem. 2011, 76, 9444-9451. doi:10.1021/jo201822k

29. Solladie, G.; Bauder, C.; Rossi, L. J. Org. Chem. 1995, 60, 7774-7777. doi:10.1021/jo00129a018

30. Ghosh, A. K.; Lei, H. J. Org. Chem. 2000, 65, 4779-4781. doi:10.1021/jo000528m

31. Hubbs, J. L.; Heathcock, C. H. J. Am. Chem. Soc. 2003, 125, 12836-12843. doi:10.1021/ja030316h

32. Xu, S. Zhongguo Xinyao Zazhi 2006, 15, 1913.

33. George, S.; Sudalai, A. Tetrahedron Lett. 2007, 48, 8544-8546. doi:10.1016/j.tetlet.2007.09.133

34. Bonini, C.; Campaniello, M.; Chiummiento, L.; Videtta, V. Tetrahedron 2008, 64, 8766-8772. doi:10.1016/j.tet.2008.06.094

35. Su, Y.; Xu, Y.; Han, J.; Zheng, J.; Qi, J.; Jiang, T.; Pan, X.; She, X. J. Org. Chem. 2009, 74, 2743-2749. doi:10.1021/jo9000146

36. Sawant, P.; Maier, M. E. Tetrahedron 2010, 66, 9738-9744. doi:10.1016/j.tet.2010.10.028

37. Xiong, F.-J.; Li, J.; Chen, X.-F.; Chen, W.-X.; Chen, F.-E. Tetrahedron: Asymmetry 2014, 25, 1205-1208. doi:10.1016/j.tetasy.2014.07.002

38. Brower, P. L.; Butler, D. E.; Deering, C. F.; Le, T. V.; Millar, A.; Nanninga, T. N.; Roth, B. D. Tetrahedron Lett. 1992, 33, 2279-2282. doi:10.1016/s0040-4039(00)74189-x

39. Rádl, S. Synth. Commun. 2003, 33, 2275-2283. doi:10.1081/scc-120021507

40. Wess, G.; Kesseler, K.; Baader, E.; Bartmann, W.; Beck, G.; Bergmann, A.; Jendralla, H.; Bock, K.; Holzstein, O.; Kleine, H.; Schnierer, M. Tetrahedron Lett. 1990, 31, 2545-2548. doi:10.1016/0040-4039(90)80121-2

41. Reddy, M. S. N.; Reddy, B. K.; Reddy, C. K.; Kumar, M. K.; Rajan, S. T.; Reddy, M. S. Orient. J. Chem. 2008, 24, 167-174.

42. Gijsen, H. J. M.; Wong, C.-H. J. Am. Chem. Soc. 1994, 116, 8422-8423. doi:10.1021/ja00097a082

43. Liu, J.; Hsu, C.-C.; Wong, C.-H. Tetrahedron Lett. 2004, 45 , 2439-2441. doi:10.1016/j.tetlet.2004.01.110

44. Öhrlein, R.; Baisch, G. Adv. Synth. Catal. 2003, 345, 713-715. doi:10.1002/adsc.200303028
45. Greenberg, W. A.; Varvak, A.; Hanson, S. R.; Wong, K.; Huang, H.; Chen, P.; Burk, M. J. Proc. Natl. Acad. Sci. U. S. A. 2004, 101, 5788-5793. doi:10.1073/pnas.0307563101

46. Müller, M. Angew. Chem., Int. Ed. 2005, 44, 362-365. doi:10.1002/anie.200460852

47. Guo, Z.; Chen, Y.; Goswami, A.; Hanson, R. L.; Patel, R. N. Tetrahedron: Asymmetry 2006, 17, 1589-1602. doi:10.1016/j.tetasy.2006.05.027

48. Sun, F.; Xu, G.; Wu, J.; Yang, L. Tetrahedron: Asymmetry 2007, 18, 2454-2461. doi:10.1016/j.tetasy.2007.09.026

49. Dong, L.; Geng, C.; Jiao, P. J. Org. Chem. 2015, 80, 10992-11002. doi:10.1021/acs.joc.5b02035

50. Han, X.; Dong, L.; Geng, C.; Jiao, P. Org. Lett. 2015, 17, 3194-3197. doi:10.1021/acs.orglett.5b00826

51. Jiang, M.; Feng, L.; Feng, J.; Jiao, P. Org. Lett. 2017, 19, 2210-2213. doi:10.1021/acs.orglett.7b00558

52. Curran, D. P. J. Am. Chem. Soc. 1983, 105, 5826-5833. doi:10.1021/ja00356a021

53. Song, Z. J.; Zhao, M.; Desmond, R.; Devine, P.; Tschaen, D. M.; Tillyer, R.; Frey, L.; Heid, R.; Xu, F.; Foster, B.; Li, J.; Reamer, R.; Volante, R.; Dolling, U. H.; Reider, P. J.; Okada, S.; Kato, Y.; Mano, E. J. Org. Chem. 1999, 64, 9658-9667. doi:10.1021/jo991292t

54. Zhao, M.; Li, J.; Mano, E.; Song, Z.; Tschaen, D. M.; Grabowski, E. J. J.; Reider, P. J. J. Org. Chem. 1999, 64, 2564-2566. doi:10.1021/j0982143y

55. Jiang, X.; Zhang, J.; Ma, S. J. Am. Chem. Soc. 2016, 138, 8344-8347. doi:10.1021/jacs.6b03948

56. Kim, J.-I.; Lee, S.-K.; Jahng, Y. Arch. Pharmacal Res. 1994, 17, 161-165. doi:10.1007/bf02974252

57. Hagiwara, H.; Kon-no, M.; Nakano, T.; Uda, H. J. Chem. Soc., Perkin Trans. 1 1994, 2417. doi:10.1039/p19940002417

58. Jefferies, P. R.; Gengo, P. J.; Watson, M. J.; Casida, J. E. J. Med. Chem. 1996, 39, 2339-2346. doi:10.1021/jm950712d

59. Karisalmi, K.; Koskinen, A. M. P. Synthesis 2004, 1331-1342. doi:10.1055/s-2004-822389

60. Tarur, V. R.; Sathe, D. G.; Rao, N. M.; Bhopalkar, R.; Mahajan, U. S. Process for the preparation of fluvastatin sodium and intermediates. PCT Int. Appl. WO 2007023503 A1, March 1, 2007.

61. Renata, H.; Zhou, Q.; Baran, P. S. Science 2013, 339, 59-63. doi:10.1126/science.1230631

62. Renata, H.; Zhou, Q.; Dünstl, G.; Felding, J.; Merchant, R. R.; Yeh, C.-H.; Baran, P. S. J. Am. Chem. Soc. 2015, 137, 1330-1340. doi:10.1021/ja512022r

63. Labeeuw, O.; Roche, C.; Phansavath, P.; Genêt, J.-P. Org. Lett. 2007, 9, 105-108. doi:10.1021/ol062631p

64. Roche, C.; Labeeuw, O.; Haddad, M.; Ayad, T.; Genêt, J.-P.; Ratovelomanana-Vidal, V.; Phansavath, P. Eur. J. Org. Chem. 2009, 3977-3986. doi:10.1002/ejoc.200900316

65. Besse, P.; Ciblat, S.; Canet, J.-L.; Troin, Y.; Veschambre, H. Tetrahedron: Asymmetry 1999, 10, 2213-2224. doi:10.1016/s0957-4166(99)00234-7

66. Koide, K.; Finkelstein, J. M.; Ball, Z.; Verdine, G. L. J. Am. Chem. Soc. 2001, 123, 398-408. doi:10.1021/ja0023377

67. Goswami, D.; Koli, M. R.; Chatterjee, S.; Chattopadhyay, S.; Sharma, A. Org. Biomol. Chem. 2017, 15, 3756-3774. doi:10.1039/c7ob00626h

68. Ma, X.; Diane, M.; Ralph, G.; Chen, C.; Biscoe, M. R. Angew. Chem., Int. Ed. 2017, 56, 12663-12667. doi:10.1002/anie.201704672 
69. Ganganna, B.; Srihari, P.; Yadav, J. S. Tetrahedron Lett. 2017, 58, 2685-2689. doi:10.1016/j.tetlet.2017.05.034

70. Ghosh, A. K.; Lei, H. J. Org. Chem. 2002, 67, 8783-8788. doi:10.1021/jo020402k

71. Das, S.; Goswami, R. K. J. Org. Chem. 2014, 79, 9778-9791. doi:10.1021/j05019798

72. Crystallographic data of 17: CCDC 1826709.

\section{License and Terms}

This is an Open Access article under the terms of the Creative Commons Attribution License

(http://creativecommons.org/licenses/by/4.0). Please note that the reuse, redistribution and reproduction in particular requires that the authors and source are credited.

The license is subject to the Beilstein Journal of Organic Chemistry terms and conditions:

(https://www.beilstein-journals.org/bjoc)

The definitive version of this article is the electronic one which can be found at: $\underline{\text { doi: } 10.3762 / \text { bjoc. } 15.179}$ 